\title{
A Unique Case of Hemolytic Uremic Syndrome Secondary to Enteropathogenic E. Coli
}

\author{
Blessie Nelson ${ }^{1}$, Angelina Hong ${ }^{1}$, Fatima Iqbal ${ }^{1}$, and Bagi Jana ${ }^{2}$ \\ ${ }^{1} \mathrm{UTMB}$ \\ ${ }^{2}$ University of Texas MD Anderson Cancer Center Division of Cancer Medicine
}

May 12, 2020

\begin{abstract}
Typical form of Hemolytic Uremic Syndrome is caused most commonly enterohemorrhagic E. coli strain O157:H71. Here we report a unique case of HUS secondary to enteropathogenic E. coli infection, with a discussion on the diagnosis of HUS and how it is distinguished from other thrombotic microangiopathies (TMAs).
\end{abstract}

\section{K E Y WO R D S}

hemolytic uremic syndrome; enteropathogenic e coli; dialysis; thrombocytopenia; hemolysis; anemia

\section{Key Clinical Message}

Causative factors of HUS due to infection are not limited to classic EHEC and Shigella infection. Understanding the effects of EPEC related HUS and its complications is imperative for early diagnosis and treatment to mitigate long term sequelae.

\section{Background :}

Hemolytic uremic syndrome (HUS) is a potentially life-threatening hematologic disorder that presents with the triad of microangiopathic anemia (MAHA), thrombocytopenia and acute renal failure secondary to vascular damage. The typical form of HUS (tHUS) is caused by an E. coli or Shigella infection, most commonly enterohemorrhagicE. coli (EHEC) strain O157:H7 $7^{1}$ Atypical HUS (aHUS) is caused by a genetic mutation causing abnormal complement activation. Enteropathogenic E. coli (EPEC) is a well-known cause of diarrhea in pediatric patients who live in developing countries, but is a rare cause of adult diarrhea, and has not been documented as a cause of HUS ${ }^{2}$. Here we report a unique case of HUS secondary to EPEC infection, with a discussion on the diagnosis of HUS and how it is distinguished from other thrombotic microangiopathies (TMA).

\section{Case Report :}

A 64-year-old female with a history of early stage, hormone positive breast cancer (treated with partial mastectomy in January 2019 and hormone therapy) presented with weakness, fatigue, nausea, vomiting, and watery, non-bloody diarrhea that started within 48 hours after eating restaurant food. In the emergency department, max temperature was $37.4\left(99.3^{\circ} \mathrm{F}\right)$, heart rate was 156 , and blood pressure was $95 / 63$. She was alert and oriented to person, place, time and situation. Her white blood cell count (WBC) was 21,430/uL, hemoglobin (HGB) $14.8 \mathrm{~g} / \mathrm{dL}$, platelet count (PLT) 227,000/uL, potassium $3.5 \mathrm{mEq} / \mathrm{L}$, lactate $8.68 \mathrm{mg} / \mathrm{dL}$, creatinine $2.2 \mathrm{mg} / \mathrm{dL}$, BUN $37 \mathrm{mg} / \mathrm{dL}$, and creatine kinase (CK) 10,846 U/L. She was diagnosed with hypovolemic shock and acute kidney injury secondary to rhabdomyolysis. She was given aggressive IV hydration 
with empiric antibiotics and was sent to the ICU for higher level of care. Over the next 5 days, she developed severe thrombocytopenia and anemia, reaching a nadir PLT count of 50 and HGB of 5.0. She was anuric and transitioned from continuous renal replacement therapy to conventional hemodialysis. Complement levels were low during this acute stress state. Blood smear showed the presence of many schistocytes and reticulocytosis (Figure 1), and labs (Table 1) confirmed hemolysis. Cold agglutinin test, paroxysmal nocturnal hemoglobinuria markers, antinuclear antibody and direct antibody testing were negative. Fecal pathogen polymerase chain reaction (PCR) testing was positive for EPEC. ADAMTS13 activity level was decreased at $32 \%$ (normal is greater than $67 \%$ ), but did not meet thrombotic thrombocytopenic purpura's (TTP) diagnostic criteria (below 5-10\%); hence, plasmapheresis therapy was not performed. Atypical HUS genetic panel was negative, and $\mathrm{C} 1$ esterase inhibitor level was within normal limits.

Clinical improvement began after steroid therapy. She was started on IV methylprednisolone $1 \mathrm{mg} / \mathrm{kg} /$ day for 12 days. She then transitioned to prednisone $60 \mathrm{mg}$ daily, and tapered the dose down by $20 \mathrm{mg}$ each week. After she completed week 3 of prednisone therapy ( $20 \mathrm{mg}$ daily), the dose was reduced to $10 \mathrm{mg}$ daily for the final week. Mentation improved, and repeat blood smear showed significantly fewer schistocytes compared to before. Her CBC and CMP values recovered, as did hemolysis labs (Table 1). She was on hemodialysis for a total of 5 weeks. She can now live independently, but residual symptoms include moderate fatigue, weakness, and decreased cognition.

\section{Discussion :}

Documented cases of typical HUS in adult patients are very rare, and usually are caused by O157:H7 strains of $E$. coli that can be cultured and/or detected by stool studies that detect the organism's Shiga-like toxins $\operatorname{stx} 1 / \mathrm{stx} 2^{3,4}$. This case is a rare example of HUS in an adult patient whose fecal pathogen PCR was positive for EPEC rather than EHEC.

EPEC produces the watery diarrhea seen in this patient case, whereas EHEC causes bloody diarrhea. EPEC produces an attaching and effacing lesion (A/E) upon the epithelial surface of the small and/or large bowel. It then utilizes a type III secretion system to inject virulence factors into host cells, and a type IV bundle forming pilus to establish microcolonies. It is hypothesized that EPEC causes watery diarrhea by disrupting the absorptive surfaces of the intestinal microvilli ${ }^{5}$. EHEC also forms A/E lesions on the intestinal epithelium, as it shares the same chromosomal pathogenicity island as EPEC. However, what distinguishes EHEC is the production of Shiga-like toxin, which binds to endothelial cells that express Gb3 and enables diffuse spread of the toxin to various cells (including renal glomerular endothelium) that express Gb3. Moreover, EHEC causes bloody diarrhea but EPEC does not because the A subunit of EHEC's Shiga-like toxin prevents protein synthesis and triggers apoptosis of the host cells ${ }^{6}$.

Typical HUS, atypical HUS and TTP are all causes of MAHA, but each have distinct mechanisms. tHUS is caused by EHEC's verotoxins orShigella 's enterotoxins, which cause vascular damage (specifically to the glomerular endothelium) and thereby increase platelet adhesion and promote microthrombi formation ${ }^{7}$. In contrast, aHUS is unrelated to E. coli or Shigellainfection and is instead caused by abnormal regulatory genes of the complement pathway. TTP is caused by antibodies disrupting the ADAMTS13 enzyme, resulting in the accumulation of von Willebrand factor multimers that cause MAHA. Another type of HUS that does not fit into the typical or atypical categories is secondary HUS, which may be caused by streptococcal infection or other acquired sources of complement dysregulation such as pregnancy, chemotherapy, malignancy, glomerular disorders, or autoimmune diseases. If the patient has no genetic abnormalities in the complement pathway, no identifiable causes of secondary HUS, and normal ADAMTS13 activity, then the TMA is idiopathic HUS ${ }^{8}$.

Where on the complex spectrum of HUS does this patient's case belong? She did not have an EHEC or Shigella infection and no Shiga or Shiga-like toxins were identified, so her TMA cannot be diagnosed as tHUS. On the other hand, with no genetic abnormality in complement regulation, the patient does not meet the criteria for aHUS either. Since the suspected cause of her TMA is EPEC, her case is best described as HUS secondary to EPEC infection. Although the possible mechanisms of EPEC-related HUS have not yet been 
elucidated, tHUS, aHUS, and TTP are all ultimately consequences of complement system hyperactivation; thus, it is feasible that EPEC's effect on the host systemic inflammatory and complement pathways could also incite HUS despite lacking EHEC's Shiga-like toxins ${ }^{9}$.

\section{Conclusion:}

HUS has many diverse presentations and etiologies, some of which are still poorly understood. Whereas HUS typically occurs in the pediatric population and is caused by EHEC, this case illustrates how HUS can also be found in adult patients and may be caused by other organisms such as EPEC. A thorough workup is required to identify HUS as the diagnosis and rule out other potential causes of MAHA and thrombocytopenia. Recognizing the different presentations and causes of HUS in adult patients is essential for conducting appropriate diagnostic studies and patient care.

Tables and Figures:

\begin{tabular}{|c|c|c|c|c|}
\hline & Admission & Day before treatment & After 12 days of high-dose IV steroids & Discharge \\
\hline Labs & Day 1 & Day 5 & Day 17 & Day 22 \\
\hline WBC & 21,430 & 42,160 & 5,490 & 10,360 \\
\hline HGB (g/dL) & 14.8 & 5.0 & 8.8 & 9.7 \\
\hline PLT & 227 & 66 & 385 & 200 \\
\hline $\mathrm{Cr}(\mathrm{mg} / \mathrm{dL})$ & 2.20 & 3.94 & 2.42 & 2.28 \\
\hline BUN (mg/dL) & 37 & 100 & 26 & 23 \\
\hline CK (U/L) & 10,846 & NA & NA & NA \\
\hline $\mathrm{LDH}(\mathrm{U} / \mathrm{L})$ & NA & 3,399 & 515 & NA \\
\hline Reticulocyte & NA & 36.0 & 34.9 & NA \\
\hline Haptoglobin (mg/dL) & NA & $<6$ & 163 & NA \\
\hline
\end{tabular}

Table 1. Lab values during hospital course. The patient initially presented with leukocytosis and elevated BUN and Cr. As she acutely developed anemia and thrombocytopenia in the context of MAHA, she was started on high-dose IV methylprednisolone after day 5. After 12 days of treatment, she was stable and hemolysis labs significantly improved.

\section{CONFLICT OF INTEREST}

None declared.

\section{AUTHOR CONTRIBUTIONS}

AA: wrote the manuscript. AM: cared for the patients, and

edited and revised the manuscript

\section{Conflict of Interest}

None declared

\section{Author Contributions}

All authors contributed equally to this manuscript.

\section{References}

1. Salvadori M, Bertoni E. Update on hemolytic uremic syndrome: diagnostic and therapeutic recommendations. World J Nephrol . 2013; 2(3): 56-76.

2. Hartland EL, Leong JM. Enteropathogenic and enterohemorrhagic E. coli : ecology, pathogenesis and evolution. Front Microbio . 2013; 3:15. 
3. Kouzy R, Alawieh R, Sukhon F, Temraz S. A case of typical hemolytic uremic syndrome in an adult. Cureus . 2018; 10(9): e3289.

4. Chinchilla-Lopez P, Cruz-Ramon V, Ramirez-Perez O, Mendez-Sanchez N. Gastroenteritis in an adult female revealing hemolytic uremic syndrome: case report. World J Gastroenterol . 2018; 24(6):763-766.

5. Hu J, Torres AG. Enteropathogenic E. coli : foe or innocent bystander? Clin Microbiol Infect . 2015; 21(8): 729-734.

6. Nguyen Y, Sperandio V. Enterohemorrhagic E. coli pathogenesis.Front Cell Infect Microbiol . 2012; 2:90.

7. Yau JW, Teoh H, Verma S. Endothelial cell control of thrombosis.BMC Cardiovasc Disord . 2015; 15:30.

8. Nester CM, Thomas CP. Atypical hemolytic uremic syndrome: what is it, how is it diagnosed, and how is it treated? Hematology . 2012(1); 617-625.

9. Noris M, Mescia F, Remuzzi G. STEC-HUS, atypical HUS and TTP are all diseases of complement activation. Nat Rev Nephrol . 2012; 8:622-633.

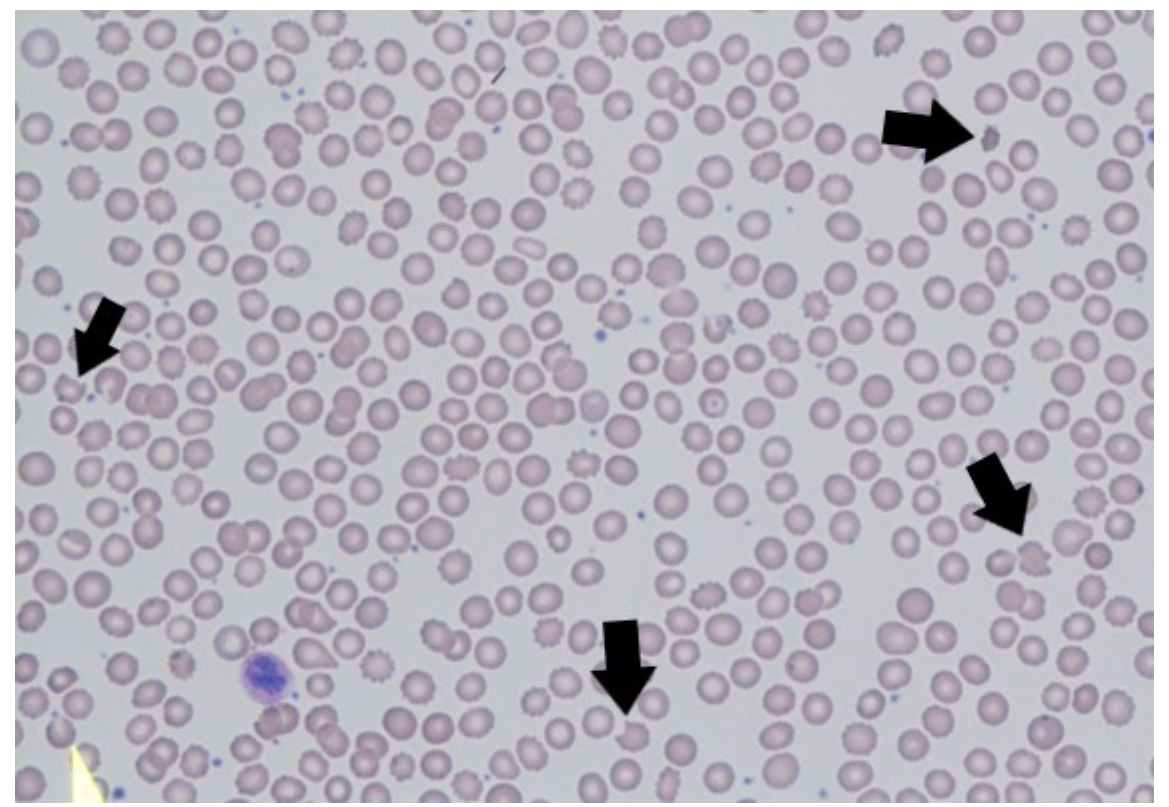

